\title{
A NOTE ON THE SPACE $L_{p}^{*}$
}

\section{J. SCHWARTZ}

The object of the following note is to give an alternate proof of the fact that the conjugate space of the Lebesgue space $L_{p}$ is the Lebesgue space $L_{p^{\prime}}$, if $p>1$, even if these spaces are constructed on a non- $\sigma$-finite measure space. The result fails if $p=1$. This result has recently been proved by $\mathrm{E}$. J. McShane. ${ }^{1}$

In what follows, where $X$ denotes a Banach space, $X^{*}$ will denote its conjugate space. If $x \in X,|x|$ will denote the norm of $x$. The symbol $(S, F, m)$ will denote a measure space, that is, a set $S$, a $\sigma$-field $F$ of its subsets, and a positive, completely additive, perhaps infinite, function $m$ defined for the sets in $F$.

Let $n$ be an integer greater than or equal to $1, p$ a real number greater than 1 . Let $p^{\prime}$ be defined by $1 / p+1 / p^{\prime}=1$. Let $\delta_{j}^{l}$ be the Kronecker $\delta$-function.

1. Let $a_{1}, \cdots, a_{n}$ all be greater than 0 . Define $R\left(n, p, a_{1}, \cdots, a_{n}\right)$ to be the Banach space of $n$-uples $y=\left[y_{i}\right]$, where the norm is defined by

$$
|y|=\left\{\sum_{i=1}^{n} a_{i}\left|y_{i}\right|^{p}\right\}^{1 / p} .
$$

Note that $\left|y+y^{\prime}\right|=\left\{\sum\left|a_{i}^{1 / p}\left(y_{i}+y_{i}^{\prime}\right)\right|^{p}\right\}^{1 / p} \leqq|y|+\left|y^{\prime}\right|$ follows from Minkowski's inequality; and that all the other requirements that $R\left(n, p, a_{1}, \cdots, a_{n}\right)$ must satisfy in order to be a Banach space are as evidently true.

Now let $x^{*} \in R^{*}\left(n, p, a_{1}, \cdots, a_{n}\right)$. If $x^{*}\left(\delta_{j}^{i}\right)=k_{i}$, it is evident that $x^{*}(y)=\sum_{i=1}^{n} k_{i} y_{i}$. If $x^{*} \neq 0$, we may note, putting

$$
v=\left[\left|\frac{k_{i}}{a_{i}}\right|^{p^{\prime}-1} \overline{\arg \left(k_{i}\right)}\right], \quad 1 \leqq i \leqq n,
$$

where $\arg (x)$ is the argument of the complex quantity $x$, that

$$
\left|x^{*}\right| \geqq \frac{\left|x^{*}(v)\right|}{|v|}=\frac{\left.\left|\sum_{i=1}^{n} k_{i}\right| \frac{k_{i}}{a_{i}}\right|^{p^{\prime}-1} \overline{\arg \left(k_{i}\right)} \mid}{\left\{\sum_{i=1}^{n} a_{i}\left(\left|\frac{k_{i}}{a_{i}}\right|^{p^{\prime}-1}\right)^{p}\right\}^{1 / p}}
$$

Received by the editors March 15, 1950.

${ }^{1}$ Proceedings of the American Mathematical Society vol. 1 (1950) pp. 402-408. 


$$
\begin{aligned}
& =\frac{\sum_{i=1}^{n} a_{i}^{1-p^{\prime}}\left|k_{i}\right|^{p^{\prime}}}{\left\{\sum_{i=1}^{n} a_{i}\left|\frac{k_{i}}{a_{i}}\right|^{p^{\prime}}\right\}^{1 / p}} \\
& =\left\{\sum_{i=1}^{n} a_{i}^{1-p^{\prime}}\left|k_{i}\right|^{p^{\prime}}\right\}^{1 / p^{\prime}} .
\end{aligned}
$$

If $x^{*}=0$, the same inequality is trivial. From Theorem 1 below, the converse inequality will follow immediately.

2. Now let $L_{p}(S, F, m)$ be the $L_{p}$ space on the measure space $S$, that is, the set of measurable functions $f(w)$ defined for $w \in S$ such that

$$
|f|=\left\{\int_{S}|f(w)|^{p} d m\right\}^{1 / p}<\infty .
$$

Consider for the moment a fixed subset $A$ of $S$ such that $m(A)<\infty$, and let $B \subseteq A$. Note that if $K_{B}(w)$ is the characteristic function of the set $B$, and $x^{*} \in L_{p}^{*}, x^{*}\left(K_{B}\right)$ is a completely additive and absolutely continuous set function. Thus, as long as $B \subseteq A$, we may write, by the theorem of Radon-Nikodym,

$$
x^{*}\left(K_{B}\right)=\int_{B} t_{A}(w) d m=\int_{S} t_{A}(w) K_{B}(w) d m .
$$

Here $t_{A}(w)$ vanishes outside $A$, while if $m\left(A^{\prime}\right)<\infty$ also, $t_{A}(w)=t_{A^{\prime}}(w)$ almost everywhere on the intersection of $A$ and $A^{\prime}$.

3. Now let $A$ be as above, and let $A \supseteq A_{i}, m\left(A_{i}\right)>0$, and let the $A_{i}$ be disjoint; let $G$ be the set of all functions constant on each of the $A_{i}$ and vanishing elsewhere, and let $w_{i} \in A_{i}$. Note that $G$, as a subset of $L_{p}$, is isometrically isomorphic to $R\left(n, p, m\left(A_{1}\right), \cdots, m\left(A_{n}\right)\right)$, $f$ in $G$ corresponding to $\left(f\left(w_{1}\right), \cdots, f\left(w_{n}\right)\right)$. If $f \in G, x^{*}(f)=\sum_{i=1}^{n} f\left(w_{i}\right)$ $\int_{A_{i}} t_{A}(w) d m$, so that, since the norm of $x^{*}$ as an element of $L_{p}^{*}$ is at least as great as its norm as an element of $G^{*}$, we may conclude, by point (1)

$$
\left|x^{*}\right| \geqq\left(\sum_{i=1}^{n} m\left(A_{i}\right)^{1-p^{\prime}}\left|x^{*}\left(K_{A_{i}}\right)\right|^{p^{\prime}}\right)^{1 / p^{\prime}} .
$$

Define $t_{A}(m, w)$ by: R.P. $\left(t_{A}(n, w)\right)=\min (k / n, n)$ if $0 \leqq k / n$ $\leqq$ R.P. $\left(t_{A}(w)\right)<(k+1) / n$; R.P. $\left(t_{A}(n, w)\right)=\max (-k / n,-n)^{-i f}$ $0 \leqq k / n \leqq \mathrm{R}$.P. $\left(-t_{A}(w)\right)<(k+1) / n$; similar inequalities hold for the 
imaginary parts. Then $\lim _{n \rightarrow \infty} t_{A}(n, w)=t_{A}(w)$. Let $A_{i}^{n}, 1 \leqq i \leqq W_{n}$, be the sets of nonzero measure on which $t_{A}(n, w)$ is constant and nonzero. On $A_{i}^{n}$ we have

$$
\left|\operatorname{R.P} .\left(\int_{A_{i}^{n}} t_{A}(w) d m\right)\right| \geqq \mid \text { R.P. }\left(t_{A}\left(n, w_{i}^{n}\right)\right)\left|m\left(A_{i}^{n}\right)\right|,
$$

where $w_{i}^{n} \in A_{i}^{n}$; a similar equation holds for the imaginary parts. Thus,

$$
\left|\int_{A_{i}^{n}} t_{A}(w) d m\right| \geqq\left|t_{A}\left(n, w_{i}^{n}\right)\right| m\left(A_{i}^{n}\right),
$$

since the absolute value of a quantity is the square-root square sum of its real and imaginary parts. From equation [*] we have

$$
\begin{aligned}
\left|x^{*}\right| & \geqq\left(\sum_{i=1}^{w_{n}} m\left(A_{i}^{n}\right)^{1-p^{\prime}} m\left(A_{i}^{n}\right)^{p^{\prime}}\left|t_{A}\left(n, w_{i}^{n}\right)\right|^{p^{\prime}}\right)^{1 / p^{\prime}} \\
& =\left(\int_{S}\left|t_{A}(n, w)\right|^{p^{\prime}} d m\right)^{1 / p^{\prime}}
\end{aligned}
$$

and, applying Fatou's lemma, we obtain $\left|x^{*}\right| \geqq\left(\int_{S}\left|t_{A}(w)\right|^{p^{\prime}} d m\right)^{1 / p^{\prime}}$. Now put $H(A)=\int_{S}\left|t_{A}(w)\right|^{p^{\prime}} d m$, and let $U \leqq\left|x^{*}\right|^{p^{\prime}}$ be l.u.b. $H(A)$, $A$ ranging over the sets where $m(A)<\infty$. Choose $A_{1}, A_{2}, \cdots$ in such a way that $\lim _{n \rightarrow \infty} H\left(A_{n}\right)=U$; where, since $H(A)$ is an increasing function of $A$, we may suppose $A_{1} \subseteq A_{2} \subseteq \ldots$. Let $T=\cup_{i=1}^{\infty} A_{i}$, and let $A_{0}$ be such that $H\left(A_{0}\right)>0$. Then $A_{0}$ cannot be disjoint from $T$, for since $H(A)$ is finitely additive, this would imply $\lim _{n \rightarrow \infty} H\left(A_{n} \cup A_{0}\right)$ $=U+H\left(A_{0}\right)>U$.

Now, putting $t_{T}(w)=\lim _{n \rightarrow \infty} t_{A_{n}}(w)$, if $A_{0}$ is of finite measure, and if $K_{A_{0}}(w)$ is its characteristic function, we may note that $K_{A_{0}}$ $=K_{A_{0} \cap T}+K_{A_{0}-T}$. Since $H\left(A_{0}-T\right)=0, x^{*}\left(K_{A_{0}-T}\right)=0$. Thus, $x^{*}\left(K_{A_{0}}\right)$ $=x^{*}\left(K_{A_{0} \cap T}\right)$. Since $K_{A_{0} \cap T}=\lim _{n \rightarrow \infty} K_{A_{0} \cap A_{n}}$, and $m\left(A_{0}\right)<\infty$,

$$
\lim _{n \rightarrow \infty} \int_{S}\left|K_{A_{0} \cap T}(w)-K_{A_{0} \cap A_{n}}(w)\right|^{p} d m=0 .
$$

Hence

$$
x^{*}\left(K_{A_{0}}\right)=\lim _{n \rightarrow \infty} x^{*}\left(K_{A_{0} \cap A_{n}}\right)=\lim _{n \rightarrow \infty} \int_{S} K_{A_{0} \cap A_{n}}(w) t_{T}(w) d m .
$$

Since $\left|x^{*}\right| \geqq\left\{\int_{S}\left|t_{T}(w)\right|^{p^{\prime}} d m\right\}^{1 / p^{\prime}}$, we have

$$
x^{*}\left(K_{A_{0}}\right)=\int_{S} K_{A_{0} \cap r^{\prime}}(w) t_{T^{\prime}}(w) d m=\int_{S} K_{A_{0}}(w) t_{r^{\prime}}(w) d m,
$$


since $t_{T}(w)$ vanishes outside $T$. Thus we have verified the equation $x^{*}(f)=\int_{S} f(w) t_{T}(w) d m$ for all the functions of a fundamental set in $L_{p}$. Since both sides of the equation represent continuous functionals on $L_{p}$, the equation must hold identically. In this case, Holder's inequality gives $\left|x^{*}\right| \leqq\left\{\int_{S}\left|t_{T}(w)\right|^{p^{\prime}} d m\right\}^{1 / p^{\prime}}$, so that we must have equality.

Once this is established, the linearity of the correspondence $x^{*}$ $\leftrightarrow t_{T}(w)$ is clear, so that we may state the following theorem.

Theorem 1. The space $L_{p}^{*}(S, F, m)$, where $(S, F, m)$ is an arbitrary measure space, and $p>1$, is isometrically isomorphic to the space $L_{p^{\prime}}(S, F, m), 1 / p+1 / p^{\prime}=1$. The isomorphism $x^{*} \leftrightarrow t(w)$ is determined by the formula

$$
x^{*}(f)=\int_{S} f(w) t(w) d m .
$$

4. The following example is due to T. Botts. Consider the measure space $\left(S^{*}, F^{*}, m^{*}\right)$, where $S^{*}$ is the open interval $(0,1)$; where $F^{*}$ is the $\sigma$-field consisting of the (finite or) denumerable subsets of the interval and their complements; and where $m^{*}(A)$ is the cardinality of $A$. It may be seen that $L_{1}\left(S^{*}, F^{*}, m^{*}\right)$ consists of those functions which vanish outside a denumerable set, and whose remaining values form an absolutely convergent series. Thus, if $f(x) \in L_{1}, x f(x) \in L_{1}$

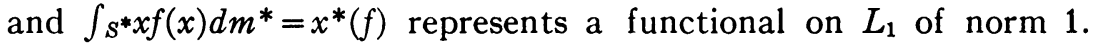
Since this functional takes on a nondenumerable set of distinct values on the family of characteristic functions of points, it cannot be of the form $\int_{S^{*}}(x) f(x) d m^{*}$, where $t(x)$ is measurable, since any measurable function must be constant except for a denumerable set.

5. For $L_{1}(S, F, m)$ the result may be stated in the following theorem.

TheOREM 2. Let $J(S, F, m)$ be the family of all absolutely continuous, countably additive, complex-valued set functions $\mu$ defined on the sets in $F$ of finite measure, satisfying the additional condition that

$$
|\mu|=\underset{0<m(A)<\infty}{\operatorname{l.u.b}} \frac{|\mu(A)|}{m(A)}<\infty .
$$

Then $J$, with the norm indicated, forms a Banach space isometrically isomorphic to $L_{1}^{*}$. The isomorphism is determined by the formula $x^{*}(f)=\int_{S} f(w) d \mu$.

Proof. If $x^{*} \in L_{1}^{*}$, it is evident that $x^{*}\left(K_{B}\right)=\mu(B)$ defines an 
absolutely continuous, countably additive set function for the sets in $F$ of finite measure, while

$$
\left|x^{*}\right| \geqq \frac{\left|x^{*}\left(K_{B}\right)\right|}{\left|K_{B}\right|}=\frac{|\mu(B)|}{m(B)}
$$

Conversely, if $\mu$ is such a function, and $|\mu(B)| / m(B) \leqq M$, and $f \in L_{1}, f$ is the limit, in the norm of $L_{1}$, of a sequence of countablyvalued functions $f_{n}=\sum_{i=1}^{\infty} a_{i}^{n} K_{B i}^{n}$; and we may put $x^{*}(f)=\lim _{n \rightarrow \infty}$ $\sum_{i=1}^{\infty} a_{i}^{n} \mu\left(B_{i}^{n}\right)$. This limit will exist and be unique only if each Cauchy sequence in $L_{1}$ of the form ${ }^{2} f_{n}=\sum_{i=1}^{\infty} a_{i}^{n} K_{B_{i}^{n}}$ corresponds to a Cauchy sequence $\sum_{i=1}^{\infty} a_{i}^{n} \mu\left(B_{i}^{n}\right)$. But

$$
\begin{aligned}
\left|\sum_{i=1}^{\infty} a_{i}^{n} \mu\left(B_{i}^{n}\right)-\sum_{j=1}^{\infty} a_{j}^{n} \mu\left(B_{j}^{n}\right)\right| & =\left|\sum_{i, j=1}^{\infty}\left(a_{i}^{n}-a_{j}^{m}\right) \mu\left(B_{i}^{n} \cap B_{j}^{m}\right)\right| \\
& \leqq M \sum_{i, j=1}^{\infty}\left|a_{i}^{n}-a_{j}^{n}\right| m\left(B_{i}^{n} \cap B_{j}^{m}\right) \\
& =M\left|f_{n}-f_{m}\right| .
\end{aligned}
$$

It may now be seen that a functional $x^{*}$ is defined, that $x^{*}\left(K_{B}\right)$ $=\mu(B)$, and that $\left|x^{*}\right|=\mu$.

Using the alternate definition of the integral as the limit of the integrals of countably-valued functions converging almost uniformly to a given function, forming a Cauchy sequence in $L_{1}(\mu)$, and observing that almost uniform convergence with respect to the measure $m$ implies almost uniform convergence with respect to $\mu$, we see that the measure and functional are related by $x^{*}(f)=\int_{s} f(w) d \mu$. Since all the required linearity relations are obvious, the theorem is proved.

6. Theorem 1 may be used to extend the following result, which is well known in the classical case.

THEOREM 3. Let $(S, F, m)$ be a measure space, and let $\mu$ be a finitely additive complex-valued function, defined for the sets of $F$ of finite measure. Then in order.that there exist a function $g(w)$ in $L_{p}, p>1$, such that $\mu(A)=\int_{A} g(w) d m$, it is necessary and sufficient that.

$$
\text { l.u.b. } \sum_{i=1}^{n}\left|\mu\left(A_{i}\right)\right|{ }^{p} m\left(A_{i}\right)^{1-p}<\infty,
$$

where $A_{1}, \cdots, A_{n}$ ranges over all finite disjoint collections of sets of $F$ of finite measure.

2 To avoid formal difficulty we may introduce a 0 coefficient and suppose $\bigcup_{i \rightarrow 1}^{\infty} B$ $=S$. The $B_{i}$ are of course supposed to be disjoint. 
Proof. Let us put

$$
x^{*}\left(\sum_{i=1}^{n} a_{i} K_{A_{i}}\right)=\sum_{i=1}^{n} a_{i} \mu\left(K_{A_{i}}\right) .
$$

Then $x^{*}$ is a linear function (as is evident from the finite additivity of $\mu$ ) defined on a dense set of $L_{p^{\prime}}$. This will be extendable to a bounded function defined on all of $L_{p^{\prime}}$, which by Theorem 1 is of the form

$$
x^{*}(f)=\int_{S} f(w) g(w) d m
$$

if and only if $\mu(A)$ is of the form $\mu(A)=\int_{A} g(w) d m$. By a well known theorem, a condition necessary and sufficient for this extension is

$$
\infty>M=\text { l.u.b. } \frac{\left|x^{*}\left(\sum_{i=1}^{n} a_{i} K_{A_{i}}\right)\right|}{\left|\sum_{i=1}^{n} K_{A_{i}}\right|} \text {. }
$$

Without loss of generality, we may assume that the $A_{i}$ are disjoint; in this case, the definition of the quantities involved gives

$$
M=\text { l.u.b. } \frac{\left|\sum_{i=1}^{n} a_{i} \mu\left(A_{i}\right)\right|}{\left\{\sum_{i=1}^{n}\left|a_{i}\right| p^{\prime} m\left(A_{i}\right)\right\}^{1 / p^{\prime}}} .
$$

The remark at the end of (1) gives

$$
M=\text { l.u.b. }\left\{\sum_{i=1}^{n}\left|\mu\left(A_{i}\right)\right| p_{m}\left(A_{i}\right)^{1-p}\right\}^{1 / p},
$$

from which the theorem is evident.

YALE UNIVERSITY 